\title{
Etmopterus Lantern Sharks Use Coelenterazine as the Substrate for Their Luciferin-luciferase Bioluminescence System
}

\section{Daichi Yano}

Chubu University

José Paitio

Chubu University

Hiromitsu Endo

Kochi University

Yuichi Oba ( $\square$ yoba@isc.chubu.ac.jp )

Chubu University

\section{Gaku Mizuno}

Chubu University

\section{Research Article}

Keywords: lantern shark genus Etmopterus, deep-sea bioluminescent fishes, coelenterazine, luciferinluciferase reaction

Posted Date: March 18th, 2021

DOl: https://doi.org/10.21203/rs.3.rs-311009/v1

License: (c) (1) This work is licensed under a Creative Commons Attribution 4.0 International License. Read Full License 


\section{Abstract}

The lantern shark genus Etmopterus is a group of deep-sea bioluminescent fishes. They emit blue light mainly from the ventral body surface, and the primary biological function is considered to be for camouflage by counterillumination. In this study, we detected both coelenterazine and coelenterazinespecific luciferase activity in the ventral photophore tissues. The results suggested that bioluminescence in lantern sharks is produced using coelenterazine as the substrate for the luciferin-luciferase reaction.

\section{Background}

Bioluminescence in cartilaginous fish has been reported in species of the squaliform families Dalatiidae, Etmopteridae, and Somniosidae ${ }^{1,2}$. They have numerous tiny photophores mainly throughout the ventral body surface, and its biological function is considered to be counterillumination, a strategy for camouflage from predators by cloaking their silhouette and making them indistinguishable from environmental downwelling light ${ }^{3,4}$. Some of these species also possess photophores on the dorsal surface, fins and dorsal spines, and the light emission is suggested to have as an aposematic function or used for intraspecific communication ${ }^{5,6}$.

Coelenterazine, 6-(4-hydroxyphenyl)-2-[(4-hydroxyphenyl)methyl]-8-(phenylmethyl)-7H-imidazo[1,2a]pyrazin-3-one, is a compound used as luciferin or chromophore of photoprotein in a wide range of marine bioluminescent organisms, including members of the phyla Radiozoa, Cercozoa, Porifera, Ctenophora, Cnidaria, Chaetognatha, Mollusca, Arthropoda, and Chordata ${ }^{7,8}$. Several coelenterazinedependent luciferase or photoprotein genes have been isolated from ctenophores, Aequorea jelly, Renilla sea pansy, Pholas bivalves, Sthenoteuthis squid, Oplophorus shrimps and copepods, and some of these genes have been used as bio-tools in molecular biology 9,10. Among bony fishes, more than 400 species of Stomiiformes and 250 species of Myctophiformes are considered to use coelenterazine-dependent bioluminescence systems ${ }^{11-14}$, but any of their luciferases have not yet been identified. Some luminous fish species in Pempheridae, Apogonidae, and Batrachoididae, which inhabit in shallow-water areas, are known to use cypridinid luciferin"; however, with the exception of Parapriacanthus ransonneti, which use cypridinid luciferase obtained from their ostracod prey ${ }^{15}$, their luciferase have not yet been determined.

In this study, we focused on the bioluminescence system employed by deep-sea sharks in the genus Etmopterus (Squaliformes, Etmopteridae). Etmopterus is one of the most diverse groups of sharks, with approximately 40 species currently recognized, and it is considered that they are probably all bioluminescent ${ }^{3,16}$. It has been reported that their bioluminescence systems are intrinsic (not symbiotic) ${ }^{17,18}$, but the reaction mechanism has not been elucidated. Based on our biochemical and chemical analyses, we concluded that etmopterid sharks use coelenterazine as the luciferin in their luciferin-luciferase bioluminescence system.

\section{Results}


Tissue distribution of luminescence activity in three Etmopterus species. Luminescence activities of various tissues in response to coelenterazine were examined in three species of Etmopterus: E. molleri, E. pusillus, E. brachyurus. The results showed that luminescence activity was predominant in tissues from the ventral skins for them (Fig. 1). Of note, Specimens 1 and 2 (Fig. 1A and 1B) are the same species morphologically of $E$. molleri, but they have small nucleotide differences between their $\mathrm{CO}$ sequences.

Detection of coelenterazine as active fraction. A crude methanol extract of ventral skin in E. molleri was separated using an octadecylsilane (ODS) column, and the luminescence activities of the fractions with the crude buffer extract were examined. The luminescence activity was detected as a single peak (Fig. $2 \mathrm{~A}$ ), and the retention time of the active fractions matches to that of authentic coelenterazine (Fig. 2B). Liquid chromatography-electrospray ionization-mass spectrometry/mass spectrometry (LC-ESI-MS/MS) analysis of the active fraction showed the molecular and fragment ion patterns (Fig. 2C) exactly agreement with those of coelenterazine (Fig. 2D ${ }^{19}$. These results suggest that the crude methanol extract contained a compound that exhibited luciferin activity to intrinsic luciferase, and that the intrinsic luciferin is coelenterazine.

Luminescence active fraction is coelenterazine-specific enzyme. The crude buffer extract of the ventral skin in E. molleri was separated by a gel-filtration column, and the luminescence activities with crude methanol extract or authentic coelenterazine were examined. The results showed that the proteins in the crude buffer extract were separated effectively (Fig. 3A), and that the luminescence activity with crude methanol extract was detected as a single peak (Fig. 3B), the retention time for the activity peak matched that for the authentic coelenterazine (Fig. 3C). These results suggest that crude buffer extract contains a protein having luciferase activity to intrinsic luciferin, and the luciferase activity is coelenterazine specific.

Luminescence spectrum and $\mathrm{pH}$ dependence of the luciferase in E. molleri. The $\mathrm{pH}$ dependence of the luminescence activity was measured using the active fractions of the gel-filtration analysis of the crude buffer extract with authentic coelenterazine. The results showed that the optimum $\mathrm{pH}$ was around 7.0 (Fig. 4A). The luminescence spectrum at pH 7.0 showed an asymmetric single-peaked curve with a tail extending into the longer wavelength region, typical for those in the most bioluminescence organisms (Fig. 4B). The peak was at $460 \mathrm{~nm}$, which corresponds to blue in human vision.

Heat and detergent susceptibility. The crude buffer extract from the ventral skin of E. molleri was treated by heat or sodium dodecyl sulfate (SDS) to test the enzyme susceptibility. The results showed that the luminescence activity was dramatically lost by the treatments (Fig. $4 \mathrm{C}$ and $4 \mathrm{D}$ ). These results suggest that the luminescence activity in the buffer extract is attributed to protein and was denatured under nonphysiological conditions like usual enzymes.

\section{Discussion}

We extracted the luciferin activity by methanol and the luciferase activity by neutral buffer from lantern sharks, the genus Etmopterus. The luminescence activities of luciferase with coelenterazine were 
predominant in ventral skin compared to other tissues (dorsal skin, muscle, and eye) for three species of Etmopterus, E. molleri, E. pusillus, and E. brachyurus. These results are consistent with in vivo observations that the ventral light emission is much greater than dorsal light emission in Etmopterus species $^{6}$. We then examined the luminescence mechanism in this genus using ventral tissues of $E$. molleri, as this species was collected most abundantly at Suruga Bay.

Identification of the luciferin molecule and luciferase protein is the most essential for understanding the bioluminescence mechanism of luminous organism being studied. In this regard, the Nobel laureate Osamu Shimomura stated "in studying bioluminescence reactions, it is crucially important to use the purified forms of the components necessary for light emission" as this avoids erroneous interpretations ${ }^{9}$. In the present study, therefore, we alternatively performed reciprocal analyses of the luciferin and luciferase using chromatographically separated fractions of luciferase and luciferin, respectively. The analyses confirmed that the luciferase activity in the buffer extract was coelenterazine-specific, and that the luciferin activity in the methanol extract was specific to coelenterazine-dependent luciferase. Mass spectrometry confirmed that the active fraction in the methanol extract is chemically identical to coelenterazine. The enzymatic activity was denatured both by heat and SDS treatments. The optimum pH of the observed luminescence activity was near physiological conditions, which is in contrast to the heterologous chemiluminescence activity observed for human albumin with coelenterazine at a highly alkaline $\mathrm{pH}^{20}$, suggesting that the luminescence activity in the shark skin extract is characteristic of a natural enzyme. The molecular size of the luciferase estimated by gel-filtration was 78-92 kDa. By further chromatographic separations, the luciferase protein can be purified.

The luminescence spectrum of the luciferase fraction with coelenterazine was peaked at $460 \mathrm{~nm}$ under neutral pH conditions. In vivo spontaneous luminescence spectra of the etmopterid shark species have been reported at wavelength of $476 \mathrm{~nm}$ in Etmopterus splendidus, $477 \mathrm{~nm}$ in E. molleri, and $486 \mathrm{~nm}$ in E. spinax ${ }^{4}$. The reason for the difference between our in vitro results and those obtained in that study is uncertain, but it may be due to genetic differences among the specimens or some effect of the colored pigment or reflectors in photophores.

Our findings contrast with those of a previous report suggesting no free-form of coelenterazine and no coelenterazine-dependent luciferase activities in the photophore tissues of Etmopterus spinax ${ }^{21}$. However, our findings showing that lantern sharks use coelenterazine for bioluminescence might be considered reasonable as lantern sharks inhabit the marine twilight zone and are higher up the food chain. It has been suggested that coelenterazine is biosynthesized in luminous copepods and comb jellies ${ }^{22,23}$, and that other luminous organisms generally obtained it through their diet, mainly by feeding on copepods or other organisms that contain coelenterazine ${ }^{24}$. During the dissection of the specimens, we found a variety of prey items in the stomach contents of E. molleri, including myctophid lanternfishes and unknown crustaceans. In analyses of $E$. spinax stomach contents, a variety of coelenterazine-dependent animals, such as Oplophorus and Pasiphaea shrimps, Maurolicus fish (Stomiiformes) and unidentified myctophid fishes, were also detected ${ }^{21,25}$. It is also known that some non-luminous fishes accumulated 
coelenterazine $^{26}$. We consider that Etmopterus lantern sharks obtained coelenterazine for bioluminescence from fishes and crustaceans diets; they also obtained coelenterazine from their diets, such as copepods. In E. spinax, in vivo luminescence from ventral photophores was also detected in the late embryonic stage ${ }^{27}$, suggesting that coelenterazine is transferred vertically from the mother.

Bioluminescence in lantern sharks is considered to have originated in the Cretaceous Period ${ }^{1}$, which is coincidental with the origin of other coelenterazine-dependent fishes, Stomiiformes and Myctophiformes ${ }^{28}$. Identification of the luciferase gene and elucidation of the coelenterazine-transport system in lantern sharks, as well as in stomiiform and myctophiform fishes, might be helpful for understanding the evolution of deep-sea bioluminescence, how it appears to have occurred simultaneously in multiple fish lineages that inhabited deep-sea environment of the Cretaceous.

\section{Materials}

Shark samples. The specimens of Etmopterus were obtained from the by-caught of commercial bottom trawls at Suruga Bay, Japan in 2019 and 2020. All specimens were frozen at $-20{ }^{\circ} \mathrm{C}$ after capture by T. A. Worldmedia (Shizuoka, Japan). The scientific name was identified by DNA analysis and morphological characteristics. The voucher specimens were deposited in the BSKU fish collection at Kochi University, Japan (E. molleri, BSKU 129578, 323 mm TL; E. pusillus, BSKU 129579, 201 mm TL; E. brachyurus, BSKU 129580, $280 \mathrm{~mm} \mathrm{TL}$ ). For DNA analysis, a partial region of the cytochrome $c$ oxidase subunit I gene (COI) was amplified and sequenced. Briefly, total DNA was extracted from muscle tissue using Lysis Buffer for PCR (Takara, Shiga, Japan) and Proteinase K (Takara). Polymerase chain reaction (PCR) was performed using the primer set Fish-F1 and Fish-R2 ${ }^{29}$ and Tks Gflex DNA polymerase (Takara, Japan). The PCR product was directly sequenced at Macrogen Inc. (Tokyo, Japan), and the sequence was deposited in the GenBank/ENA/DDBJ database (accession numbers, now depositing).

Tissue distribution analysis. Each tissue sample (ventral skin, dorsal skin, dorsal body muscle, and eye ball) was dissected from a single defrosted specimen. Approximately $0.5 \mathrm{~g}$ of each tissue sample was homogenized in $500 \mathrm{~mL}$ of cold extraction buffer (20 mM Tris-HCl, $10 \mathrm{mM}$ EDTA, pH7.4) using a plastic pestle, and centrifuged at 7,197 ' $\mathrm{g}$ for $30 \mathrm{~min}$. The extraction buffer $(90 \mathrm{~mL})$ containing $1.18 \mathrm{mM}$ of synthetic coelenterazine (JNC Corporation, Tokyo, Japan) was injected into $30 \mathrm{~mL}$ aliquot of the supernatant, and the luminescence activity was measured for 2 min using a 96-well luminometer Centro LB960 (Berthold, Bad Wildbad, Germany). The accumulated value (RLU, relative light unit) was normalized by the protein concentration of the extract, which was measured using a Protein Assay Kit (Bio-Rad, Hercules, CA).

Extraction and HPLC separation of luciferin. Dissected ventral skin samples of E. molleri were homogenized in cold methanol ( $\mathrm{mL} /$ wet weight $\mathrm{g}$ ) containing a 1:100 volume of $1 \mathrm{M}$ dithiothreitol using a homogenizer Ultra-Turrax T25 (IKA-Werke, Staufen, Germany), and centrifuged at 7,197 ' $g$ for 30 min. The supernatant was collected as the crude methanol extract. The crude methanol extract was desalted and concentrated using a MonoSpin C18 reversed-phase column (GL Science, Tokyo, Japan) and 
separated by high performance liquid chromatography (HPLC) (SEC System Prominence 501, Shimadzu, Kyoto, Japan) with a Cadenza CD-C18 column (2.0×75 mm, Imtakt, Kyoto, Japan). The fluorescence was detected at an excitation wavelength of $435 \mathrm{~nm}$ and an emission wavelength of $530 \mathrm{~nm}$ using a fluorescence detector RF-10AXL (Shimadzu). The mobile phase was an aqueous/methanol solution containing $0.1 \%$ formic acid, and the linear gradient of methanol was from $25 \%$ to $95 \%$ ( $2 \%$ per min). The flow rate was $0.2 \mathrm{~mL} / \mathrm{min}$. Fractions were collected at 1-min intervals.

Extraction and FPLC separation of luciferase. Dissected ventral skin samples of E. molleri was homogenized in cold buffer (20 mM Tris-HCl, $10 \mathrm{mM}$ EDTA, pH7.4) (1 mL/ wet weight g) using a homogenizer Ultra-Turrax T25, and centrifuged at 7,197' $g$ for $30 \mathrm{~min}$. The supernatant was collected as crude buffer extract. The crude buffer extract was filtrated using a membrane filter Millex-SV $5.0 \mathrm{~mm}$ (Merck, Darmstadt, Germany) and separated by fast protein liquid chromatography (FPLC) using AKTA Prime Plus (Cytiva, Uppsala, Sweden). The column for gel filtration was a HiLoad 16/600 Superdex 200 prep grade (Cytiva) at a flow rate of $1.0 \mathrm{~mL} / \mathrm{min}$; mobile phase, $20 \mathrm{mM}$ Bis-Tris- $\mathrm{HCl}, 150 \mathrm{mM} \mathrm{NaCl}, \mathrm{pH} 7.4$. Fractions were collected at 3-min intervals.

Bioluminescence assay of HPLC fractions. A $30 \mathrm{~mL}$ aliquot of crude buffer extract in $265 \mathrm{~mL}$ of $20 \mathrm{mM}$ Tris- $\mathrm{HCl}(\mathrm{pH} 7.2)$ was mixed with a $5 \mathrm{~mL}$ aliquot of each HPLC fraction, and the luminescence activity was measured for 5 min using a 96-well luminometer Centro LB960.

Bioluminescence assay of FPLC fractions. A $100 \mathrm{~mL}$ of $1 / 300$ diluted crude methanol extract or $1.18 \mathrm{mM}$ coelenterazine in $20 \mathrm{mM}$ Tris- $\mathrm{HCl}(\mathrm{pH} 7.2)$ was added into a $50 \mathrm{~mL}$ aliquot of each FPLC fraction, and the luminescence activity was measured for 5 min using a 96-well luminometer Centro LB960.

Spectral measurement.In vitro bioluminescence spectrum was measured using the active fraction of the FPLC purification. The active fraction was concentrated using a $50 \mathrm{kDa}$ cutoff filter Amicon Ultra-4 (Merck), and of $20 \mathrm{~mL}$ was mixed with $2 \mathrm{~mL}$ of $5.9 \mathrm{mM}$ coelenterazine and $278 \mathrm{~mL}$ of $20 \mathrm{mM}$ Bis-Tris-HCl $(\mathrm{pH}$ 7.0). The luminescence spectrum was measured using a fluorescence spectrophotometer FP-777W (Jasco, Tokyo, Japan) with the excitation light source turned off. The obtained raw spectrum was smoothed using a binomial method.

Mass spectrometry. LC-ESI-MS/MS analysis was performed using the positive mode with nitrogen as the collision gas (collision energy, $30 \mathrm{~V}$ ) using an API 4000 (AB SCIEX, Framingham, MA) connected to an LC800 HPLC system (GL Sciences) and a Cadenza CD-C18 column (Imtakt). The mobile phase was an aqueous/methanol solution containing $0.1 \%$ formic acid, and the linear gradient of methanol used was from $25 \%$ to $95 \%$ ( $2 \%$ per $\mathrm{min}$ ). The flow rate was $0.2 \mathrm{~mL} / \mathrm{min}$. For the product ion scan analysis, an aliquot ( $5 \mathrm{~mL}$ ) of HPLC fraction was applied and $m / z=424.0$, corresponding to the calculated $[\mathrm{M}+\mathrm{H}]^{+}$ mass value of coelenterazine, was monitored.

Heat and SDS treatment. The crude buffer extract was heat treated using heat block MG-1200 (Eyela, Tokyo, Japan) set at $98^{\circ} \mathrm{C}$ for $15 \mathrm{~min}$, or mixed with SDS (final concentration, 2\%) at room temperature $\left(18{ }^{\circ} \mathrm{C}\right)$ for $5 \mathrm{~min}$. A $90 \mathrm{~mL}$ of $1.18 \mathrm{mM}$ authentic coelenterazine was injected into a $10 \mathrm{~mL}$ aliquot of heat 
or SDS treated extract, and the luminescence activity was measured for 1 min using a 96-well luminometer Centro LB960.

\section{Declarations}

\section{Data availability}

The authors declare that all data supporting the findings of this study are available within the article. DNA sequence data are deposited in GenBank/ENA/DDBJ.

\section{Author contributions}

G.M., D.Y., and Y.O. conceived the idea of the study. Y.O. planned the study methodology. G.M., D.Y., J.P. and H.E. performed the experiments and species identifications. G.M. and D.Y. performed data analysis. G.M., D.Y., J.P., H.E. and Y.O. wrote the manuscript.

\section{Acknowledgements}

We thank Prof. Kaname Tsutsumiuchi (Chubu University) for assistance with the HPLC and mass spectrometry measurements, and Assoc. Prof. Shiro Takei (Chubu University) for reviewing the manuscript and providing critical comments. The manuscript was proofread by a professional English editing service, Forte Co. This work was supported by JST CREST (JPMJCR16N1).

\section{Competing interests}

The authors declare no competing interests.

Correspondence and requests for materials should be addressed to Y.O.

\section{References}

1. Straube, N., Li, C., Claes, J. M., Corrigan, S. \& Naylor, G. J. P. Molecular phylogeny of Squaliformes and first occurrence of bioluminescence in sharks. BMC Evol. Biol. 15, 162; 10.1186/s12862-0150446-6 (2015).

2. Duchatelet, L., Marion, R. \& Mallefet, J. A third luminous shark family: Confirmation of luminescence ability for Zameus squamulosus (Squaliformes; Somniosidae). Photochem. Photobiol. (in press).

3. Straube, N., Iglésias, S. P., Sellos, D. Y., Kriwet, J. \& Schliewen, U. K. Molecular phylogeny and node time estimation of bioluminescent lantern sharks (Elasmobranchii: Etmopteridae). Mol. Phylogenet. Evol.56, 905-917 (2010).

4. Claes, J., Nilsson, D. -E., Straube, N., Collin, S. P. \& Mallefet, J. Iso-luminance counterillumination drove bioluminescent shark radiation. Sci. Rep.4, 4328; 10.1038/srep04328 (2014). 
5. Claes, J. M. \& Mallefet, J. Ontogeny of photophore pattern in the velvet belly lantern shark, Etmopterus spinax. Zoology112, 433-441 (2009).

6. Duchatelet, L., Pinte, N., Tomita, T., Sato, K. \& Mallefet, J. Etmopteridae bioluminescence: dorsal pattern specificity and aposematic use. Zool. Lett.5, 9; 10.1186/s40851-019-0126-2 (2019).

7. Haddock, S. H. D., Moline, M. A. \& Case, J. F. Bioluminescence in the sea. Annu. Rev. Mar. Sci.2, 443493 (2010).

8. Martini, S., Schultz, D. T., Lundsten, L. \& Haddock, S. H. D. Bioluminescence in an undescribed species of carnivorous sponge (Cladorhizidae) from the deep sea. Front. Mar. Sci.7, 576476; 10.3389/fmars.2020.576476 (2020).

9. Shimomura, O. Bioluminescence: Chemical Principles and Methods (World Scientific, 2006).

10. Krasitskaya, V. V., Bashmakova, E. E. \& Frank, L. A. Coelenterazine-dependent luciferase as a powerful analytical tool for research and biomedical applications. Int. J. Mol. Sci.21, 7465; 10.3390/ijms21207465 (2020)

11. Shimomura, O. Widespread occurrence of coelenterazine in marine bioluminescence. Comp. Biochem. Physiol.65B, 435-437 (1980).

12. Campbell, A. K. \& Herring, P. J. Imidazolopyrazine bioluminescence in copepods and other marine organisms. Mar. Biol.104, 219-225 (1990).

13. Mallefet, J. \& Shimomura, O. Presence of coelenterazine in mesopelagic fishes from the strait of Messina. Marine Biol. 124, 381-385 (1995).

14. Thompson, E. M. \& Rees, J. -F. Origins of luciferins: ecology of bioluminescence in marine fishes in Biochemistry and molecular biology of fishes Vol. 4 (eds. Hochachka, P. W. \& Mommsen, T. P.) 435466 (Elsevier, 1995).

15. Bessho-Uehara, M., Yamamoto, N., Shigenobu, S., Mori, H., Kuwata, K. \& Oba, Y. Kleptoprotein bioluminescence: Parapriacanthus fish obtain luciferase from ostracod prey. Sci. Adv.6, eaax4942; 10.1126/sciadv.aax4942 (2020).

16. Vásquez, V. E., Ebert, D. A. \& Long, D. J. Etmopterus benchleyi n. sp., a new lanternshark (Squaliformes: Etmopteridae) from central eastern Pacific Ocean. J. Ocean Sci. Found. 17, 43-55 (2015).

17. Renwart, M., Delroisse, J., Claes, J. M. \& Mallefet, J. Ultrastructural organization of lantern shark (Etmopterus spinax Linnaeus, 1758) photophores. Zoomorphology133, 405-416 (2014).

18. Duchatelet, L., Delroisse, J., Flammang, P., Mahillon, J. \& Mallefet, J. Etmopterus spinax, the velvet belly lanternshark, does not use bacterial luminescence. Acta Histochem.121, 516-521 (2019).

19. Oba, Y., Tsuduki, H., Kato, S., Ojika, M. \& Inouye, S. Identification of the luciferin-luciferase system and quantification of coelenterazine by mass spectrometry in the deep-sea luminous ostracod Conchoecia pseudodiscophora. ChemBioChem5, 1495-1499 (2004).

20. Vessel, N. et al. Enzymatic activity of albumin shown by coelenterazine chemiluminescence. Luminescence27, 234-241 (2011). 
21. Renwart, M. \& Mallefet, J. First study of the chemistry of the luminous system in a deep-sea shark, Etmopterus spinax Linnaeus, 1758 (Chondrichthyes: Etmopteridae). J. Exp. Mar. Biol. Ecol.448, 214219 (2013).

22. Oba, Y., Kato, S., Ojika, M. \& Inouye, S. Biosynthesis of coelenterazine in the deep-sea copepod, Metridia pacifica. Biochem. Biophys. Res. Commun.390, 684-688 (2009).

23. Bessho-Uehara, M. et al. Evidence for de novo biosynthesis of the luminous substrate coelenterazine in ctenophores. iScience23, 101859; 10.1016/j.isci.2020.101859 (2020).

24. Oba, Y. \& Schultz, D. T. Eco-Evo Bioluminescence on Land and in the Sea in Bioluminescence: Fundamentals and Applications in Biotechnology Vol. 1, Advances in Biochemical Engineering/Biotechnology 144 (eds. Thouand, G. \& Marks, R.) 3-36 (Springer, 2014).

25. Neiva, J., Coelho, R. \& Erzini, K. Feeding habits of the velvet belly lanternshark Etmopterus spinax (Chondrichthyes: Etmopteridae) off the Algarve, southern Portugal. J. Mar. Biol. Ass. UK86, 835-841 (2006).

26. Shimomura, O. Presence of coelenterazine in non-bioluminescent marine organisms. Comp. Biochem. Physiol.86B, 361-363 (1987).

27. Duchatelet, L., Claes, J. M. \& Mallefet, J. Embryonic expression of encephalopsin supports bioluminescence perception in lanternshark photophores. Mar. Biol.166, 21; 10.1007/s00227-0193473-9 (2019).

28. Davis, M. P., Sparks, J. S. \& Leo Smith, W. Repeated and widespread evolution of bioluminescence in marine fishes. PLOS ONE11, e01555154; 10.1371/journal.pone.0155154 (2016).

29. Ward, R. D., Zemlak, T. S., Innes, B. H., Last, P. R. \& Hebert, P. D. N. DNA barcoding Australia's fish species. Phil. Trans. R. Soc. B360, 1847-1857 (2005).

\section{Figures}
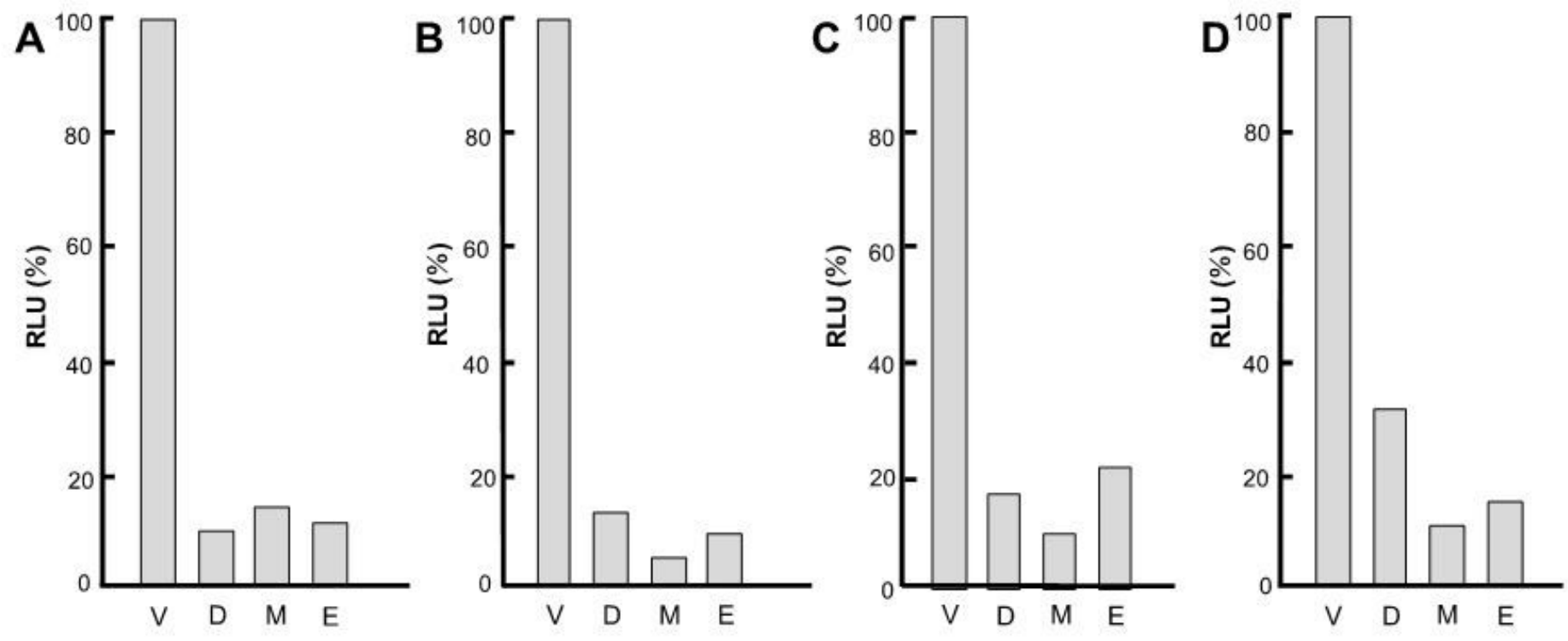
Figure 1

Tissue distributions of the luminescence activity with coelenterazine in Etmopterus spp. (A) Etmopterus molleri Specimen 1, (B) E. molleri Specimen 2 (C) Etmopterus pusillus, (D) Etmopterus brachyurus. V, ventral skin; $D$, dorsal skin; $M$, muscle; $E$, eye. The values are the mean of triplicate measurements and shown as percentage to values obtained for ventral skin.
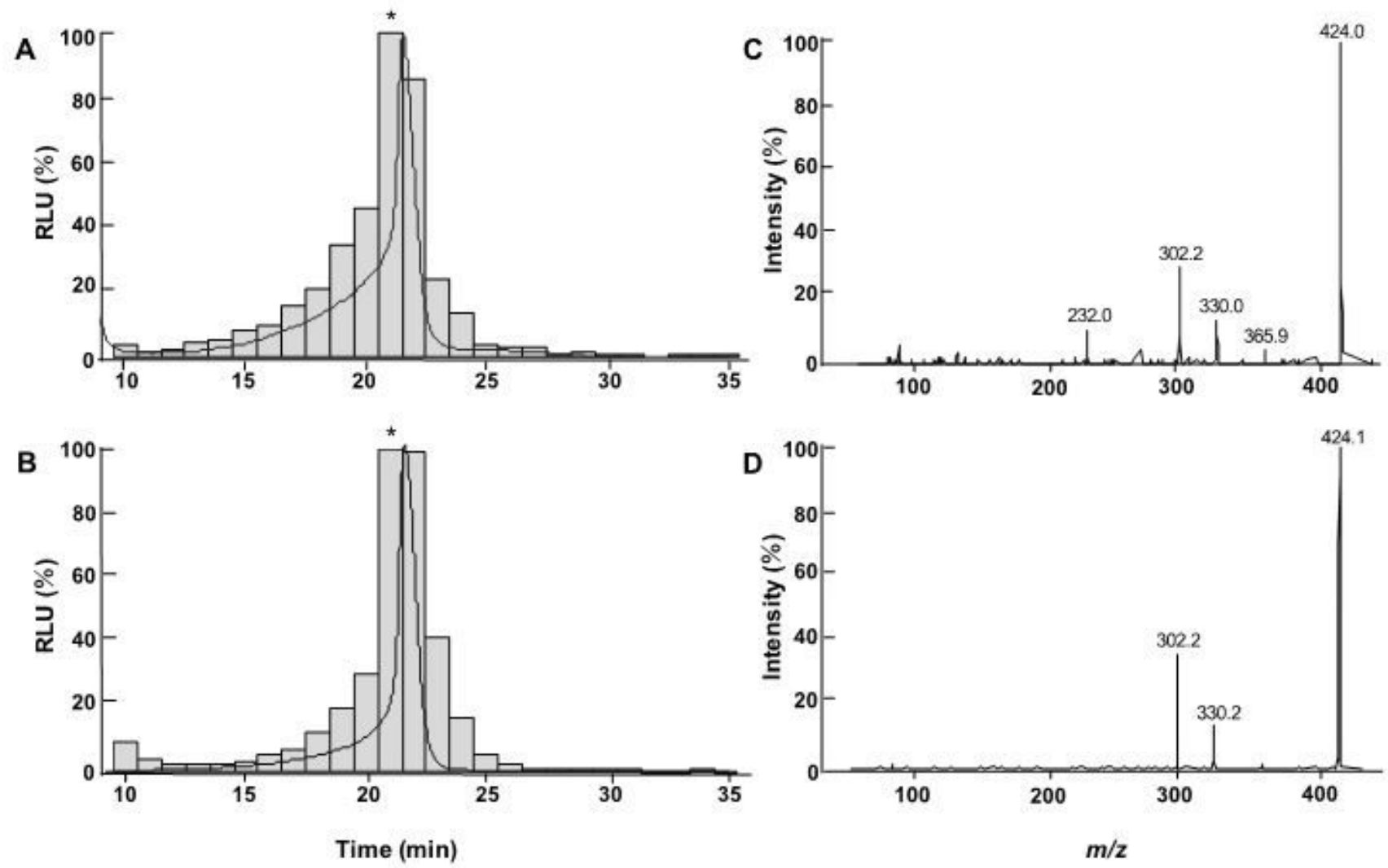

Figure 2

Luminescence activity and LC-ESI-MS/MS analyses of the HPLC fraction of the crude methanol extract from ventral skin in $E$. molleri $(A, C)$ and authentic coelenterazine $(B, D)$. (A, B) Lines represent fluorescence emission; bars represent the luminescence activity of the fraction. Asterisks represent the fraction used for the LC-ESI-MS/MS analyses. Panels $C$ and $D$ show the results of the product scan analysis for the fraction indicated by the asterisk of $A$ and $B$, respectively. 

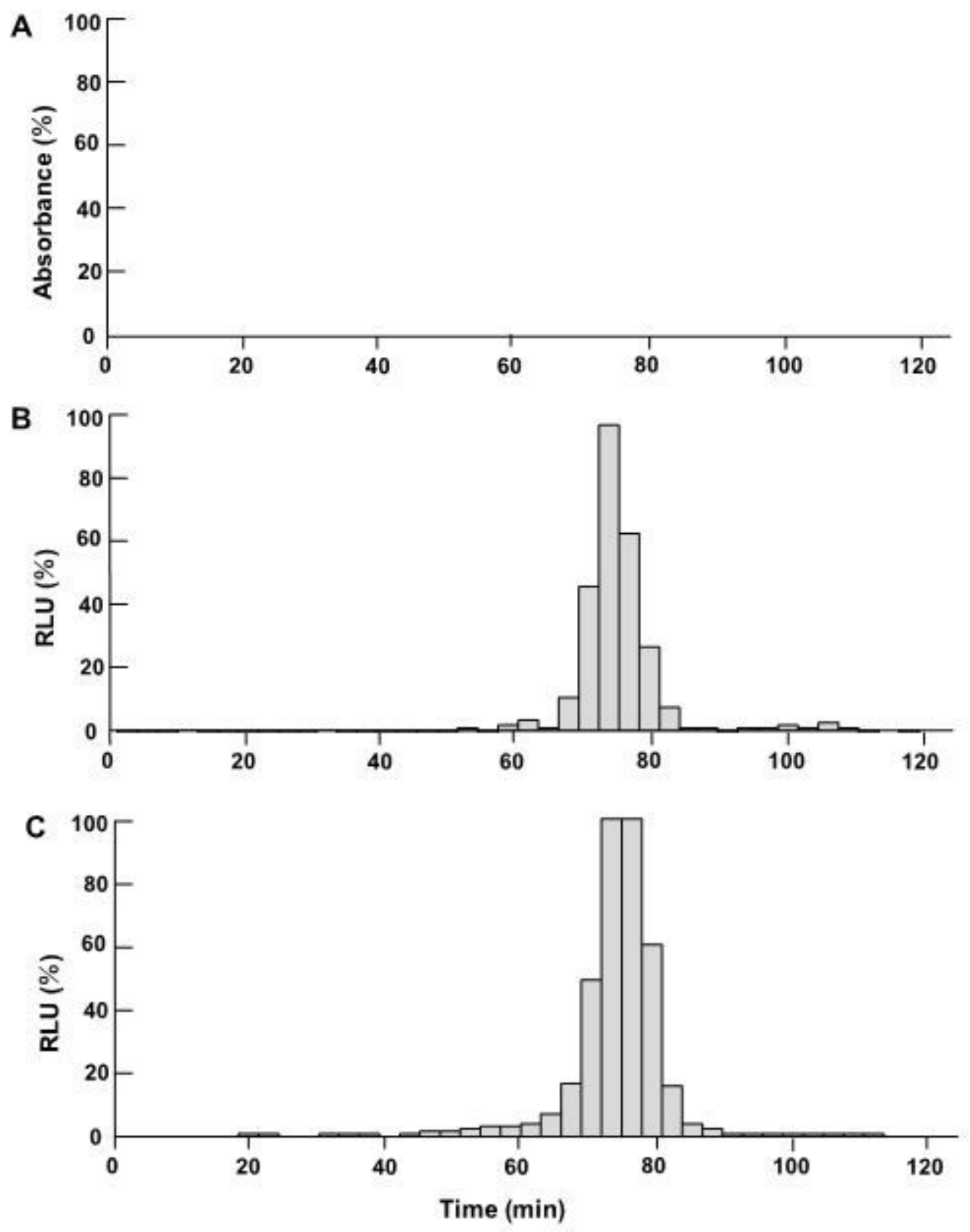

\section{Figure 3}

FPLC analyses of the crude buffer extract of ventral skin in E molleri. (A) UV detection at $280 \mathrm{~nm}$. (B) Luminescence activity of the fraction with the crude methanol extract. (C) Luminescence activity of the fraction with authentic coelenterazine. 
A

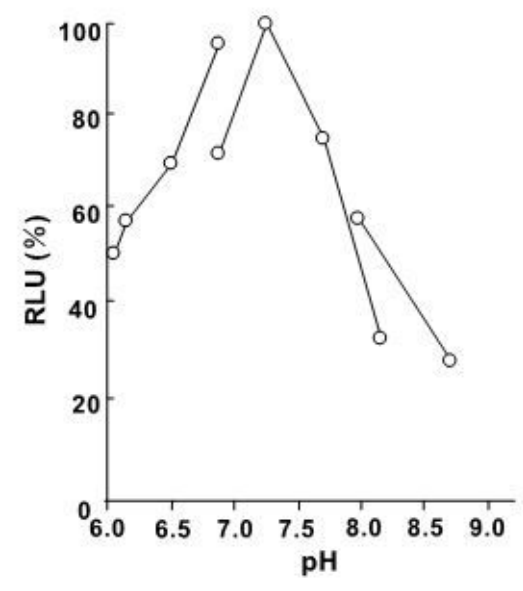

B

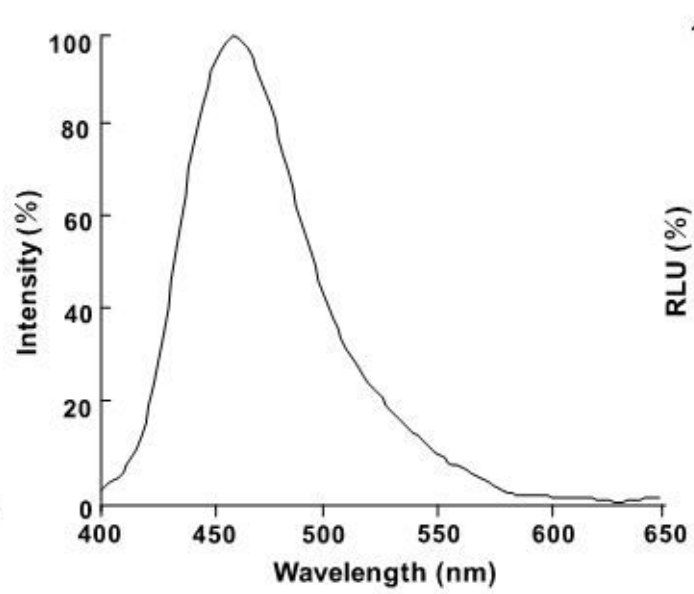

C

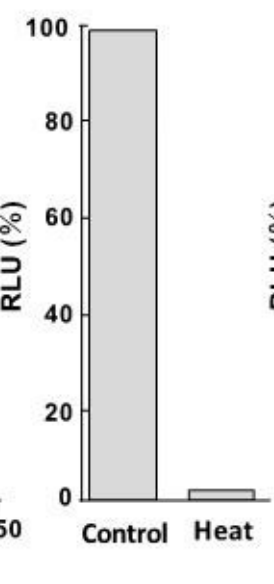

D

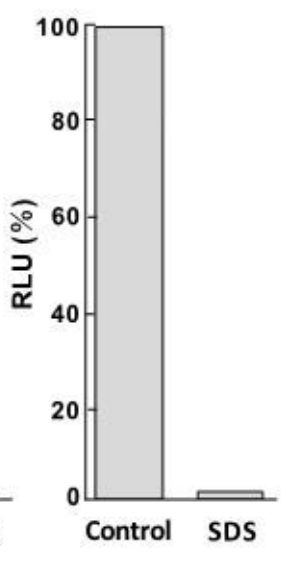

\section{Figure 4}

Enzymatic characteristics of the FPLC-purified luciferase with coelenterazine. (A) pH-dependent activity profiles. For $\mathrm{pH}$ adjustment, Bis-Tris- $\mathrm{HCl}$, Tris- $\mathrm{HCl}$, and glycine-NaOH buffers were used for $\mathrm{pH}$ 6-7, $\mathrm{pH}$ 78.5, and pH8.5-9.5, respectively. (B) Luminescence spectrum at pH 7.0. at $18{ }^{\circ} \mathrm{C}(\mathrm{C})$ Luminescence activity after the treatment at $18{ }^{\circ} \mathrm{C}$ for $15 \mathrm{~min}$ (Control) or $98^{\circ} \mathrm{C}$ for $15 \mathrm{~min}$ (Heat). (D) Luminescence activity after the treatment without (Control) and with $2 \%$ SDS (SDS) at $18{ }^{\circ} \mathrm{C}$ for $5 \mathrm{~min}$. 